OPEN ACCESS

Edited by:

Bruce Campbell,

University of Melbourne,

Australia

Reviewed by:

Felix Ng,

Austin Health, Australia

Nawaf Yassi,

University of Melbourne,

Australia

*Correspondence:

Qiaoshu Wang

qwang624@139.com

tThese authors have contributed equally to this work.

Specialty section: This article was submitted

to Stroke,

a section of the journal

Frontiers in Neurology

Received: 20 August 2017

Accepted: 23 October 2017

Published: 07 November 2017

Citation:

Liu L, Wu B, Zhao J, Cao Y,

Dedhia N, Caplan LR and Wang $Q$

(2017) Computed Tomography

Perfusion Alberta Stroke Program

Early Computed Tomography Score

Is Associated with Hemorrhagic

Transformation after Acute

Cardioembolic Stroke.

Front. Neurol. 8:591.

doi: 10.3389/fneur.2017.00591

\section{Computed Tomography Perfusion Alberta Stroke Program Early Computed Tomography Score Is Associated with Hemorrhagic Transformation after Acute Cardioembolic Stroke}

\author{
Lan Liu't, Bo Wu't, Jinglong Zhao ${ }^{3}$, Yanyan Cao', Nikita Dedhia', Louis R. Caplan ${ }^{5}$ \\ and Qiaoshu Wang ${ }^{1,5 *}$
}

\begin{abstract}
'Department of Neurology, Shanghai General Hospital, Shanghai Jiao Tong University School of Medicine, Shanghai, China, ${ }^{2}$ Center of Cerebrovascular Diseases, Department of Neurology, West China Hospital, Sichuan University, Chengdu, China, ${ }^{3}$ Department of Radiology, Shanghai General Hospital, Shanghai Jiao Tong University School of Medicine, Shanghai, China, ${ }^{4}$ Department of Neurology, University Hospitals of Cleveland, Case Western Reserve University, Cleveland, $\mathrm{OH}$, United States, ${ }^{5}$ Department of Neurology, Beth Israel Deaconess Medical Center, Harvard Medical School, Boston, MA, United States
\end{abstract}

Alberta Stroke Program Early Computed Tomography (CT) score (ASPECTS) has been applied to CT perfusion (CTP) with good interrater agreement to predict early ischemic stroke, and it can be useful in decision making in acute ischemic stroke. The aim of the present study was to assess the predictive value of CTP ASPECTS of hemorrhagic transformation $(\mathrm{HT})$ in acute cardioembolic stroke. This is a single-enter, retrospective study. All patients hospitalized with acute cardioembolic stroke from January 2008 to September 2013 were included. ASPECTS of baseline non-contrast CT, CTP maps of cerebral blood volume (CBV), cerebral blood flow, and mean transit time were collected from 52 consecutive patients with less than 12-h anterior circulation ischemic stroke. MRI scan was performed within $72 \mathrm{~h}$ of symptom onset after index stroke including $\mathrm{T}^{*}$-weighted gradient echo to identify HT. For bleeding risk assessment, CTP and diffusion-weighted imaging ASPECTS were categorized into 0-7 or 8-10. Baseline characteristics, ASPCETS scores and HT were compared. Eighteen (34.6\%) patients had HT and four (7.7\%) developed symptomatic HT. On univariate analysis, the proportion of patients with CBV-ASPECTS 0-7 was significantly higher in $\mathrm{HT}$ patients as compared to patients without $\mathrm{HT}$ (44 versus $9 \%, P=0.005$ ). CBV ASPECTS 0-7 remained independent prognostic factors for $\mathrm{HT}$ after adjustment for clinical baseline variables. CBV ASPECTS could be of value to predict HT risk after acute cardioembolic stroke and may be a quick risk assessment approach before reperfusion therapy.

Keywords: cerebral infarction, hemorrhagic transformation, computed tomography perfusion, Alberta Stroke Program Early Computed Tomography scores, cardioembolic stroke 


\section{INTRODUCTION}

Hemorrhagic transformation (HT) can cause devastating consequence of ischemic stroke, especially for cardioembolic stroke, occurring in up to $90 \%$ of patients within the first week after symptom onset (1-4). In recent endovascular therapy for ischemic stroke, computed tomography (CT) perfusion (CTP) was used to identify the ischemic penumbra in the EXTEND-IA trial, and in the MR CLEAN trial, although perfusion imaging was not used as an inclusion/exclusion criteria, CTP was done in about $65 \%$ of patients $(5,6)$. The purpose of using CTP in these studies was to exclude patients with large ischemic stroke and without salvageable ischemic tissue, and such patients have higher odds of hemorrhage or malignant edema caused by reperfusion $(7,8)$.

Alberta Stroke Program Early CT score (ASPECTS) has been applied to CTP with good interobserver variability, and CTP ASPECTS is more accurate at predicting the extent of reversible and irreversible ischemia than non-contrast CT (NCCT) $(9,10)$. Diffusion-weighted imaging (DWI) ASPECTS has been proved to be a reliable surrogate of lesion volume in patients with middle cerebral artery stroke, and an independent prognostic factor for symptomatic intracerebral hemorrhage (sICH) after thrombolysis $(11,12)$. Although automated software analysis can be used to process the CTP imaging, it is not available in many stroke centers. A technique such as ASPECTS could provide rapid, inexpensive and widely applicable assessment of early ischemic changes (EICs). Furthermore, low ASPECTS may be associated with an increased risk of HT. Thus, the present study aims to assess the value of CTP ASPECTS as a method of predicting the HT after acute cardioembolic stroke.

\section{MATERIALS AND METHODS}

\section{Participants}

We sifted all patients at the Beth Israel Deaconess Medical Center of the Harvard Medical School, Boston who met the international criteria for diagnosis of acute ischemic stroke between January 2008 and September 2013 with the approval of The Institutional Review Board. Etiologic origin of the stroke was determined according to the medical records of the patients using the Causative Classification System for Ischemic Stroke and its electronic implementation available online (https://ccs.mgh.harvard. edu/main.php) $(13,14)$.

Patients were included in the present analysis if (1) they had acute cardioembolic stroke in the anterior circulation and undergone NCCT and CTP within $12 \mathrm{~h}$ of symptom onset and (2) an MRI scan was performed within $72 \mathrm{~h}$ of symptom onset after index CTP including DWI, T2*-weighted gradient echo (GRE), and MR angiography (MRA). Patients with poor image quality, no GRE sequence, no MRA or CT angiography (CTA), posterior circulation infarction, and anterior cerebral artery infarction were excluded for further analysis. HT was defined as GRE documented hemorrhage in the ischemia occurred within 3 days after treatment onset (Figure 1) $(15,16)$. Parenchymal hematoma $(\mathrm{PH})$ was defined as hemorrhage with mass effect. sICH definition was $\mathrm{PH}$ and NIHSS worsening $\geq 4$ points
(17-19). The following clinical variables that have considered to be associated with sICH were collected for each patient: age, NIHSS score on admission, blood pressure level at admission, leukoaraiosis (LA), cerebral microbleeds (CMBs), antiplatelet or anticoagulation use before admission, CHADS2 score, INR level, and reperfusion therapy [including intravenous tissue plasminogen activator ( $t-P A)$, intra-arterial delivery of $t-P A$, and mechanical thrombectomy].

\section{Imaging}

All patients underwent NCCT, CTP, and CTA according to a standardized protocol as part of routine clinical assessment. Whole-brain NCCT was performed using $120 \mathrm{kV}, 250 \mathrm{~mA}$, and slice thickness $5 \mathrm{~mm}$. The following CTP used $40 \mathrm{ml}$ non-ionic iodinated contrast at a rate of $4 \mathrm{ml} / \mathrm{s}$ via a power injector (acquisition time $90 \mathrm{~s}, 80 \mathrm{kV}, 500 \mathrm{~mA}$ maximum), providing 16, 5-mm thick slices through the anterior circulation.

MRI scans were performed on $1.5 \mathrm{~T}$ scanner. MRI protocol included $\mathrm{T} 1$ - and T2-weighted, fluid-attenuated inversion recovery (FLAIR), axial trace DWI with $2 b$-values ( 0 and 1,000), apparent diffusion coefficient, time of flight MR angiography, and T2*-weighted GRE sequence. The MRI parameters for DWI and GRE were a slice thickness of $5 \mathrm{~mm}$ (no gap between slices) with a matrix size of $128 \times 128,24-30$ axial slices, and a field-of-view of $240 \mathrm{~mm}$. DWI (EPI spin echo) parameters were a repetition time (TR) of 4,528 ms, an echo time (TE) of $103 \mathrm{~ms}$. Other imaging parameters were as follows: GRE (TR $835 \mathrm{~ms}$; TE $26 \mathrm{~ms}$ ), FLAIR (TR 9,000 ms; TE $84 \mathrm{~ms}$ ), T1 (TR $420 \mathrm{~ms}$; TE $8.8 \mathrm{~ms}$ ), and T2 (TR 4,500 ms; TE $95 \mathrm{~ms}$ ).

\section{Image Analysis}

All ASPECTS were assessed independently by two experienced investigators, a neuroradiologist (ZJ) and a neurologist (CY) both blinded to patient identity and clinical information. A template of two axial NCCT/CTP/DWI slices with markers for the 10 regions being scores by the NCCT/CTP/DWI ASPECTS was offered. Scoring of NCCT, CTP and DWI was done separately.

Non-contrast CT ASPECTS were accessed for focal parenchymal low attenuation or swelling (Figure 1) (20). All raters evaluated the CTP images in the same order: cerebral blood volume (CBV), cerebral blood flow (CBF), and mean transit time (MTT). The raters scored ASPECTS region as abnormal if there was a relative reduction in $\mathrm{CBV}$ or $\mathrm{CBF}$ maps as dark blue, or relative increase in MTT maps as red (Figure 1). Relative threshold values for irreversible lesion and penumbra are $\mathrm{CBV}<40 \%, \mathrm{CBF}<30 \%$, and MTT $>145 \%$ compared with the normal contralateral hemisphere $(5,9,21-23)$. For MRI, relative hyperintensity on DWI ASPECTS region was scored as abnormal $(9,24)$.

Leukoaraiosis was defined on MR FLAIR image as hyperintense supratentorial white matter lesions. Fazekas scale was used both in periventricular $(0=$ absent, $1=$ caps or pencil lining, $2=$ smooth halo, and $3=$ irregular periventricular hyperintensities extending into deep white matter) and subcortical areas $(0=$ absent, $1=$ punctuate foci, $2=$ beginning confluence of foci, and $3=$ large confluent areas) $(25,26)$. LA was graded in nonischemic hemisphere and the total Fazekas score was calculated by adding the periventricular and subcortical scores. CMBs were 

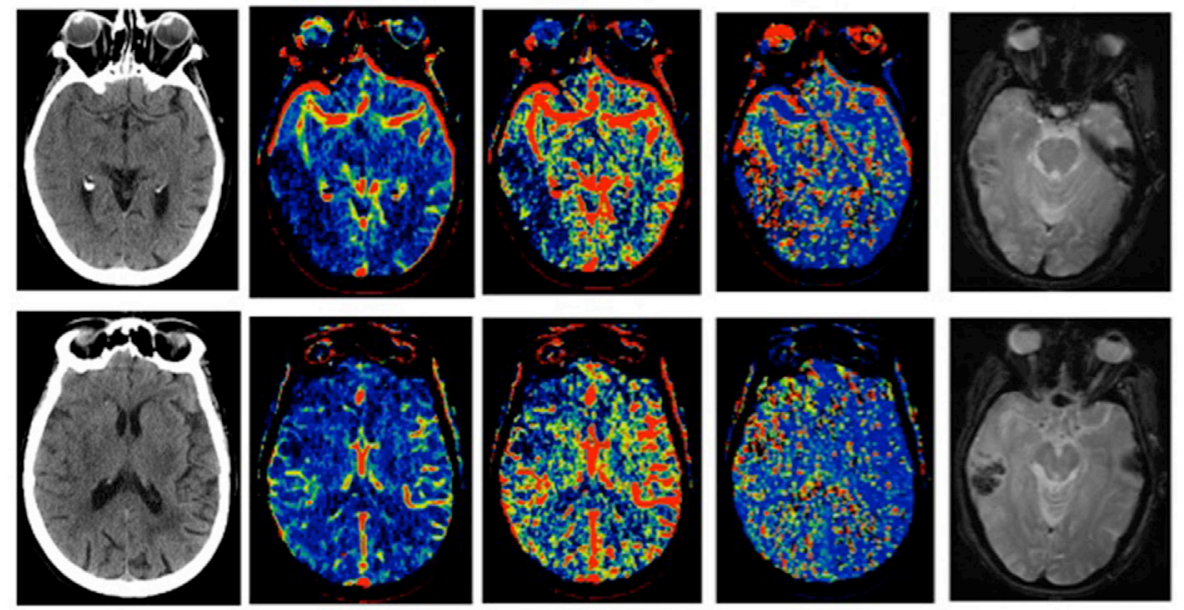

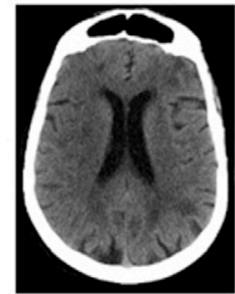

NCCT

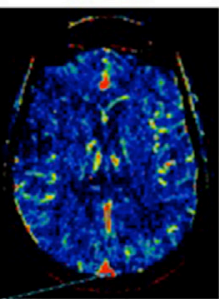

CBV

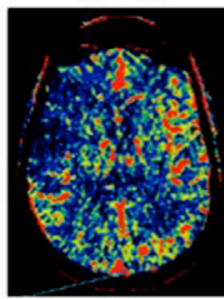

CBF

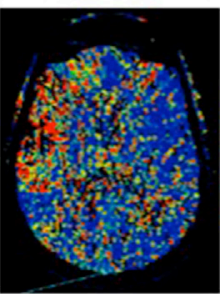

MTT

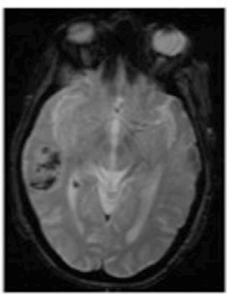

GRE

FIGURE 1 | An 87-years-old woman was imaged $3 \mathrm{~h}$ after symptom onset with left-sided weakness. Subtle loss of gray-white matter differentiation in the right insular ribbon on non-contrast computed tomography [NCCT; Alberta Stroke Program Early Computed Tomography (CT) score (ASPECTS), 9]. Reduced cerebral blood volume (CBV; ASPECTS, 5) involving the right temporal lobe and frontal lobe in the middle cerebral artery (MCA) territory and more obvious abnormality on cerebral blood flow (CBF; ASPECTS, 4) and mean transit time (MTT; ASPECTS, 4) in the right temporal lobe, frontal lobe and insular cortex. After $7 \mathrm{~h}$ of symptom onset hyperintensity on diffusion-weighted imaging (DWI) in the right temporal lobe, frontal lobe, and insular cortex and hypointensity on T2*-weighted gradient echo (GRE) was identified in the right temporal lobe.

defined as small (generally $2-5 \mathrm{~mm}$ in diameter but up to $10 \mathrm{~mm}$ ) areas of signal void on $\mathrm{T} 2{ }^{*}$-weighted sequences (27).

To evaluate of the present of vessel occlusion, two observers (Jinglong Zhao and Yanyan Cao) independently reviewed the circle of Willis maximum intensity projection reconstructions of acute CTA and graded middle cerebral artery as proximal and distal occlusion. Follow-up MRA was also graded in the same manner.

\section{Statistical Analysis}

The mean value of ASPECTS of both observers was used for statistical analysis. Non-integral numbers of the mean ASPECTS of the two observers were uprounded for categorization (i.e., if the mean CBV-ASPECTS was 6.5, the patient was categorized in the 7-10 CBV-ASPECTS group). The patients were divided into two groups by the presence of HT at follow up GRE image. First, Mean ASPECTS on NCCT, CTP, and DWI modalities were compared with each other between groups using $t$ tests for normal distribution (Kolmogorov-Smirnov test) and the Mann-Whitney $U$ for non-normality. Second, ASPECTS were dichotomized (scores 0-7 versus scores 8-10) according to previous CT-based study $(11,28)$. Then these cut points were used as independent variables in binary logistic regression analysis to determine whether an ASPECTS was an independent prognostic factor for HT. Other independent variables considered in the regression equations were age of 78 years or younger versus older than 78 years, baseline NIHSS of 15 or less versus greater than 15, blood pressure level at admission, Fazekas scale of LA, absence versus presence of acute vessel occlusion, reperfusion therapy, and antiplatelet or anticoagulation use before admission (28). Third, Interobserver reliability was tested for ASPECTS as a continuous variable using an intraclass correlation coefficient with one-way analysis of variance. Results were considered statistically significant at the $5 \%$ level. For statistical analysis the SPSS 20.0 software (SPSS Inc.) was used. The weighted kappa statistics and the ROC analyses were calculated with MedCalc (Version 16.8.4) (24).

\section{RESULTS}

Of 61 patients suspected of having an acute cardioembolic stroke in the anterior circulation territory, 9 patients were excluded due to no GRE imaging (1 patient), poor imaging of CTP (3 patients), posterior circulation or anterior cerebral artery infarction (5 patients). Therefore, a total of 52 cardioembolic ischemic stroke patients were included in the analysis (mean age, 75 years; 33 women). The NIHSS score on admission was $10.5 \pm 7.0$ (interquartile range, 5-16). Median time from symptom onset to NCCT and CTP imaging was $5.2 \pm 4.8 \mathrm{~h}$ (interquartile 
range, 2.0-7.0 h) and from symptom onset to MRI imaging was $30.7 \pm 30.0 \mathrm{~h}$ (interquartile range, $12.0-39.5 \mathrm{~h}$ ). Of 24 patients who received reperfusion therapy, 15 patients were treated with standard dose intravenous recombinant tissue plasminogen activator (rt-PA). Seven patients received intravenous rt-PA and mechanical thrombectomy, and two patients were given intravenous and intra-arterial rt-PA. The interval time from onset to reperfusion therapy is $2.6 \pm 1.0 \mathrm{~h}$ (interquartile range, 1.8-3.0 h). Table 1 gives an overview of demographic and clinical variables between HT and non-HT patients. Patients with reperfusion therapy had a $70 \%$ risk of HT as compared to a $32 \%$ risk in patients without HT. Interobserver reliability for the assessment of ASPECTS was substantial (weighted kappa 0.723 for NCCT, 0.775 for CBV-ASPECTS, 0.778 for CBF-ASPECTS, 0.852 for MTT-ASPECTS, and 0.887 for DWI-ASPECTS).

The mean ASPECTS showed significant difference between modalities in the following order (Table 2): NCCT and $\mathrm{CBV}>\mathrm{CBF}>\mathrm{MTT}$ and DWI. Mean ASPECTS on NCCT and CBV, MTT, and DWI were similar (Table 2). CBV ASPECTS exceeded other modalities especially MTT (mean, 2.5).

Eighteen (34.6\%) patients had HT. Six patients (11.5\%) had parenchymal hemorrhage, and four (7.7\%) developed sICH. All the sICH patients were given reperfusion therapy. On univariate analysis, the proportion of patients with CBV-ASPECTS 0-7 was significantly higher in HT patients as compared to patients without HT (44 versus $9 \%, P=0.005$; Table 3 ). The proportion of patients with DWI-ASPECTS $0-7$ was $89 \%$ in HT patients and $47 \%$ in patients without HT ( $P=0.008$; Table 3$)$. Accordingly, mean DWI-ASPECTS were significant lower in HT patients as compared to patients without HT [6 (2) versus 7 (2), $P=0.005$; Table 3].

Sensitivity, specificity, positive predictive value (PPV) and negative predictive value (NPV) for HT prediction are provided in Table 4 by dichotomized imaging criteria of CBV and

\begin{tabular}{|c|c|c|c|}
\hline \multirow[t]{2}{*}{ Variables } & \multicolumn{2}{|c|}{$\begin{array}{l}\text { Hemorrhagic } \\
\text { transformation }\end{array}$} & \multirow[t]{2}{*}{$\boldsymbol{P}$} \\
\hline & Yes $(n=18)$ & No $(n=34)$ & \\
\hline Sex, male & $8(44)$ & $11(32)$ & 0.58 \\
\hline Age, $>78$ years & $7(39)$ & $20(59)$ & 0.28 \\
\hline NIHSS score $>15$ & $5(28)$ & $8(24)$ & 1.00 \\
\hline Systolic blood pressure (mm Hg) & $153(28)$ & $154(28)$ & 0.93 \\
\hline Diastolic blood pressure (mm Hg) & $78(18)$ & $82(18)$ & 0.51 \\
\hline Symptom onset to CTP (h) & $4.1(3.2)$ & $5.8(5.4)$ & 0.50 \\
\hline Symptom onset to MRI (h) & $34.2(34.9)$ & $28.9(27.4)$ & 0.67 \\
\hline Symptom onset to reperfusion therapy & $2.33(1.0)$ & $2.99(1.0)$ & 0.42 \\
\hline Antiplatelet use & $7(39)$ & $18(53)$ & 0.50 \\
\hline Anticoagulant use & $3(17)$ & $7(21)$ & 1.00 \\
\hline MCA occlusion & $15(83)$ & $24(71)$ & 0.50 \\
\hline Proximal MCA occlusion & $9(50)$ & $11(32)$ & 0.34 \\
\hline CHADS score & $2(1)$ & $2(1)$ & 0.23 \\
\hline Cerebral microbleeds & $1(6)$ & $1(3)$ & 1.00 \\
\hline INR & $1.2(0.3)$ & $1.4(0.6)$ & 0.34 \\
\hline Fazekas score & $2(2)$ & $3(2)$ & 0.70 \\
\hline Reperfusion therapy & $13(72)$ & $11(32)$ & 0.01 \\
\hline
\end{tabular}

Values are mean (SD) or n (\%), as appropriate.
DWI-ASPECTS 0-7 (versus scores 8-10). Sensitivity was low (0.44) for CBV-ASPECTS 0-7, whereas specificity was high (0.91). Oppositely, high sensitivity (0.89) and low specificity (0.53) were found for DWI-ASPECTS 0-7. PPV (0.72) was medium for CBVASPECTS 0-7, and low (0.50) for DWI-ASPECTS 0-7. NPV was medium (0.75) for CBV-ASPECTS 0-7 and high (0.90) for DWIASPECTS 0-7. Table 4 provides data on medium (0.72 and 0.68) sensitivity and specificity, low (0.54) PPV and high (0.82) NPV for HT prediction by dichotomized therapy criteria of reperfusion therapy and no use. Using a ROC analysis, the area under the curve did not show significance between CBV-ASPECTS [0.678 (95\% CI 0.534-0.801)] and DWI-ASPECTS [0.709 (95\% CI $0.567-0.827)$ ], indicating the significant predictive values of the two methods.

TABLE 2 | Comparison of the mean value of ASPECTS $(95 \% \mathrm{Cl}$ for difference between means, paired $t$-test)

\begin{tabular}{lccccc}
\hline All patients & NCCT & CBV & CBF & MTT & DWI \\
\hline Mean (SD) & $8.6(1.3)$ & $8.8(1.7)$ & $7.2(2.0)$ & $6.3(1.8)$ & $6.6(2.0)$ \\
NCCT & & $-0.9-0.4$ & $0.6-2.2^{\mathrm{a}}$ & $1.5-3.0^{\mathrm{a}}$ & $1.2-2.7^{\mathrm{a}}$ \\
CBV & & & $1.0-2.2^{\mathrm{a}}$ & $1.7-3.2^{\mathrm{a}}$ & $1.6-2.8^{\mathrm{a}}$ \\
CBF & & & & $0.3-1.3^{\mathrm{a}}$ & $-0.2-1.4$ \\
MTT & & & & & $-1.2-0.6$ \\
\hline
\end{tabular}

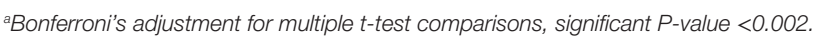
ns, not significant; Cl, confidence interval; NCCT, non-contrast computed tomography; CBV, cerebral blood volume; CBF, cerebral blood flow; MTT, mean transit time; DWI, diffusion-weighted imaging.

TABLE 3 | Comparison of the ASPECTS for HT versus non-HT, using $t$-tests and dichotomous ASPECTS cut point.

\begin{tabular}{|c|c|c|c|}
\hline \multirow[t]{2}{*}{ ASPCETS } & \multicolumn{2}{|c|}{ Hemorrhagic transformation } & \multirow[t]{2}{*}{$P$} \\
\hline & Yes $(n=18)$ & No $(n=34)$ & \\
\hline NCCT, mean value & $8(1)$ & $9(1)$ & 0.42 \\
\hline NCCT 0-7 vs. >7 & $4(22)$ & $8(24)$ & 1 \\
\hline CBV, mean value & $8(2)$ & $9(2)$ & 0.29 \\
\hline CBV 0-7 vs. >7 & $8(44)$ & $3(9)$ & 0.005 \\
\hline CBF, mean value & $7(2)$ & $7(2)$ & 0.4 \\
\hline CBF 0-7 vs. >7 & $13(72)$ & $17(50)$ & 0.21 \\
\hline MTT, mean value & $6(2)$ & $6(2)$ & 0.89 \\
\hline MTT 0-7 vs. >7 & $15(83)$ & $24(71)$ & 0.45 \\
\hline DWI, mean value & $6(2)$ & $7(2)$ & 0.005 \\
\hline DWI 0-7 vs. >7 & $16(89)$ & $16(47)$ & 0.008 \\
\hline
\end{tabular}

Values are mean (SD) or $n$ (\%), as appropriate.

NCCT, non-contrast computed tomography; CBV, cerebral blood volume; CBF, cerebral blood flow; MTT, mean transit time; DWI, diffusion-weighted imaging.

TABLE 4 | Sensitivity, specificity, positive, and negative predictive values for CBV and DWI-ASPECTS 0-7 and recanalization therapy for HT prediction.

\begin{tabular}{llcc}
\hline & CBV-ASPECTS 0-7 & DWI-ASPECTS 0-7 & $\begin{array}{c}\text { Reperfusion } \\
\text { therapy }\end{array}$ \\
\hline Sensitivity & 0.44 & 0.89 & 0.72 \\
Specificity & 0.91 & 0.53 & 0.68 \\
PPV & 0.72 & 0.5 & 0.54 \\
NPV & 0.75 & 0.9 & 0.82
\end{tabular}


In a binary logistic regression model, CBV-ASPECTS 0-7 was independently associated with HT when controlling for reperfusion therapy [odds ratio (OR) 10.26 (95\% CI 1.83-57.64, $P=0.008)]$. Likewise, DWI-ASPECTS $0-7$ was an independent risk factor in this binary logistic regression model [OR 11.61 (95\% CI 1.92-70.29, $P=0.008)$ ].

\section{DISCUSSION}

Our results indicate that CBV and DWI ASPECTS 0-7 are associated with $\mathrm{HT}$ after cardioembolic ischemic stroke.

The ASPECTS scoring system was initial designed to improve EIC detection on CT scans $(28,29)$. Although the "goldstandard" for the assessment of EIC is DWI, manual volumetry is time consuming and impractical when quick decision making is needed. On the contrary, ASPECTS DWI scores, a semiquantitative tool of the estimation of the DWI lesion size, have been shown to be of value to apply easily and quickly in the clinical setting. Previous study, performed in patients received thrombolytic treatment, demonstrated that pretreatment DWI ASPECTS 0-7 were associated with sICH and DWI AEPECTS scores correlated well with the DWI lesion volume (11). Furthermore, in accordance with previous study, our series in acute cardioembolic stroke indicates that DWI ASPECTS as a semiquantitative estimate of the DWI lesion size might predict HT with high sensitivity but low specificity. However, the DWI data in our study were acquired $30 \mathrm{~h}$ after index stroke. At this point, early decisions about thrombolytic treatment had been made.

In most institutions, CTP is still the preferred choice of rapid diagnosis of hyperacute ischemic stroke by generating maps of $\mathrm{CBV}, \mathrm{CBF}$, and MTT. Low CBV the same as DWI, is an indicator of infarction. Several studies explored the predictive value of CBV and showed that lower relative cerebral blood volume and very low cerebral blood volume were more powerful predictor of HT than other parameters of CTP in acute ischemic stroke $(30,31)$. But all those measurements of CBV need software processing and are time-consuming. The current analysis suggests that CBV ASPECTS 0-7 may predict HT risk in an easy and quick way. In our study, the mean value of NCCT and CBV ASPECTS are similar. In HT group there are more 0-7 CBV ASPECTS patients (44 versus 9\%) in comparison with no HT group, and 0-7 NCCT ASPECTS are similar between HT and no HT groups. We think it is because that $\mathrm{CBV}$ is more sensitive and specific for identifying irreversible ischemic lesion than NCCT. As Figure 1 showed, CBV has the advantage of detecting ischemic areas after acute infarction over NCCT.

Previous study has showed that CBV ASPECTS closely predicted final infarct in patients with major reperfusion, and CBF and MTT ASPECTS predicted final infarct in patients without major reperfusion (9). In our study, about 50\% patients received reperfusion therapy. MTT ASPECTS are similar to DWI ASPECTS and CBV ASPECTS are larger than final infarct ASPECTS, which suggest that most patients did not have major reperfusion, and early lower CBV ASPECTS may reflect large final necrotic area and blood-brain barrier (BBB) breakdown. Our findings demonstrate that CBV ASPECTS 0-7 have low sensitivity and high specificity, medium NPV and PPV. Given the rapid applicability and good interrater agreement, CBV ASPECTS may be favored for quick HT risk evaluation of acute cardioembolic stroke.

In our analysis, the similar ASPECTS of MTT and DWI indicate that most reperfusion therapy is ineffective, which is in accordance with previous study that delayed recanalization after acute cardioembolic stroke is an independent predictor of HT (32). Furthermore, our data show that all the four patients with sICH $(7.7 \%)$ received reperfusion therapy. The findings support the view that the duration of ischemia is an important determinant in the development of HT and delayed recanalization may cause upstream blood flow passing through impaired downstream BBB. Otherwise, proteolysis of $\mathrm{BBB}$ triggered by tPA and mechanical damage to the blood vessel endothelium due to endovascular therapy may also contribute to the HT (33).

Similar to prior studies, we have shown that CBF ASPECTS are approximate to DWI ASPECTS and CBV ASPECTS underestimate DWI volumes (34-36). Since the median time between CTP and DWI scans was $25 \mathrm{~h}$, there was likely little infarct growth in most cases. Based on previous studies, it is hard to make treatment decisions due to the high CTP measurement variability caused by poor contrast-to-noise ratios $(34,36,37)$. In those studies, the investigators used semi- or full-automated software to measure CTP lesion volume. Our findings of CTP ASPECTS measurement have shown substantial interrater reliability, which indicate a reliable and easy-to-use tool in making clinical assessment (38).

The main limitation of our study is the retrospective nature of the design. DWI ASPECTS were not performed in the hyperacute stage of stroke, which does not support the feasibility of using DWI ASPECTS in real time. In most institutions CT and CTP are still the first choice of evaluation the ischemia of stroke. Due to the higher sensitivity of CTP for detection of early ischemia as compared to $\mathrm{CT}$, it may therefore be possible that CTP ASPECTS were more reliable than CT ASPECTS. Therefore, the relationship of CBV ASPECTS and HT risk may have been established in the present analysis. Other limitations are the relatively small number of stroke patients, which was a consequence of our strict inclusion criteria.

In conclusion, our study indicates that as a widely applicable and semiquantitative methodology that does not require advanced automated software analysis, CBV ASPECTS could be of value in predicting HT risk after acute cardioembolic ischemic stroke and may be a quick risk assessment approach before reperfusion therapy. Obviously, our findings should be prospectively assessed in a large study.

\section{ETHICS STATEMENT}

This is a chart review study approved by The Institutional Review Board of the Beth Israel Deaconess Medical Center of the Harvard Medical School.

\section{AUTHOR CONTRIBUTIONS}

QW and LC had full access to all of the data in the study and take responsibility for the integrity of the data and the 
accuracy of the data analysis. Study concept and design: QW and LC. Acquisition of data: LL, QW, YC, JZ, and ND. Analysis and interpretation of data: LL, QW, and BW. Drafting of the manuscript: LL, QW, and LC. Critical revision for important intellectual content: LL, QW, BW, and LC. Study supervision: QW and LC.

\section{REFERENCES}

1. Okada Y, Yamaguchi T, Minematsu K, Miyashita T, Sawada T, Sadoshima S, et al. Hemorrhagic transformation in cerebral embolism. Stroke (1989) 20:598-603. doi:10.1161/01.STR.20.5.598

2. Jaillard A, Cornu C, Durieux A, Moulin T, Boutitie F, Lees KR, et al. Hemorrhagic transformation in acute ischemic stroke. The MAST-E study. MAST-E Group. Stroke (1999) 30:1326-32. doi:10.1161/01.STR.30.7.1326

3. Kerenyi L, Kardos L, Szasz J, Szatmari S, Bereczki D, Hegedus K, et al. Factors influencing hemorrhagic transformation in ischemic stroke: a clinicopathological comparison. Eur J Neurol (2006) 13:1251-5. doi:10.1111/ j.1468-1331.2006.01489.x

4. Larrue V, von Kummer RR, Muller A, Bluhmki E. Risk factors for severe hemorrhagic transformation in ischemic stroke patients treated with recombinant tissue plasminogen activator: a secondary analysis of the EuropeanAustralasian Acute Stroke Study (ECASS II). Stroke (2001) 32:438-41. doi:10.1161/01.STR.32.2.438

5. Campbell BC, Mitchell PJ, Kleinig TJ, Dewey HM, Churilov L, Yassi N, et al. Endovascular therapy for ischemic stroke with perfusion-imaging selection. N Engl J Med (2015) 372:1009-18. doi:10.1056/NEJMoa1414792

6. Borst J, Berkhemer OA, Roos YB, van Bavel E, van Zwam WH, van Oostenbrugge RJ, et al. Value of computed tomographic perfusion-based patient selection for intra-arterial acute ischemic stroke treatment. Stroke (2015) 46:3375-82. doi:10.1161/STROKEAHA.115.010564

7. Lansberg MG, Straka M, Kemp S, Mlynash M, Wechsler LR, Jovin TG, et al. MRI profile and response to endovascular reperfusion after stroke (DEFUSE 2): a prospective cohort study. Lancet Neurol (2012) 11:860-7. doi:10.1016/ S1474-4422(12)70203-X

8. Inoue M, Mlynash M, Straka M, Lansberg MG, Zaharchuk G, Bammer R, et al. Patients with the malignant profile within 3 hours of symptom onset have very poor outcomes after intravenous tissue-type plasminogen activator therapy. Stroke (2012) 43:2494-6. doi:10.1161/STROKEAHA.112.653329

9. Parsons MW, Pepper EM, Chan V, Siddique S, Rajaratnam S, Bateman GA, et al. Perfusion computed tomography: prediction of final infarct extent and stroke outcome. Ann Neurol (2005) 58:672-9. doi:10.1002/ana.20638

10. Lin K, Rapalino O, Law M, Babb JS, Siller KA, Pramanik BK. Accuracy of the Alberta stroke program early CT score during the first 3 hours of middle cerebral artery stroke: comparison of noncontrast CT, CT angiography source images, and CT perfusion. AJNR Am J Neuroradiol (2008) 29:931-6. doi:10.3174/ajnr.A0975

11. Singer OC, Kurre W, Humpich MC, Lorenz MW, Kastrup A, Liebeskind DS, et al. Risk assessment of symptomatic intracerebral hemorrhage after thrombolysis using DWI-ASPECTS. Stroke (2009) 40:2743-8. doi:10.1161/ STROKEAHA.109.550111

12. de Margerie-Mellon C, Turc G, Tisserand M, Naggara O, Calvet D, Legrand L, et al. Can DWI-ASPECTS substitute for lesion volume in acute stroke? Stroke (2013) 44:3565-7. doi:10.1161/STROKEAHA.113.003047

13. Ay H, Furie KL, Singhal A, Smith WS, Sorensen AG, Koroshetz WJ. An evidence-based causative classification system for acute ischemic stroke. Ann Neurol (2005) 58:688-97. doi:10.1002/ana.20617

14. Ay H, Benner T, Arsava EM, Furie KL, Singhal AB, Jensen MB, et al. A computerized algorithm for etiologic classification of ischemic stroke: the causative classification of stroke system. Stroke (2007) 38:2979-84. doi:10.1161/ STROKEAHA.107.490896

15. The National Institute of Neurological Disorders and Stroke rt-PA Stroke Study Group. Tissue plasminogen activator for acute ischemic stroke. N Engl J Med (1995) 333:1581-7. doi:10.1056/NEJM199512143332401

16. Larrue V, von Kummer R, del Zoppo G, Bluhmki E. Hemorrhagic transformation in acute ischemic stroke. Potential contributing factors in the European

\section{ACKNOWLEDGMENTS}

QW was sponsored by grant number 81371304 from the National Natural Science Foundation of China, grand number 15PJD031 from Shanghai Pujiang Program, and grand number 12ZR1424400 from the Natural Science Foundation of Shanghai.

Cooperative Acute Stroke Study. Stroke (1997) 28:957-60. doi:10.1161/01. STR.28.5.957

17. Hacke W, Kaste M, Fieschi C, Toni D, Lesaffre E, von Kummer R, et al. Intravenous thrombolysis with recombinant tissue plasminogen activator for acute hemispheric stroke. The European Cooperative Acute Stroke Study (ECASS). JAMA (1995) 274:1017-25. doi:10.1001/jama.1995.03530130023023

18. Pessin MS, Del Zoppo GJ, Estol CJ. Thrombolytic agents in the treatment of stroke. Clin Neuropharmacol (1990) 13:271-89. doi:10.1097/00002826199008000-00001

19. Furlan A, Higashida R, Wechsler L, Gent M, Rowley H, Kase C, et al. Intraarterial prourokinase for acute ischemic stroke. The PROACT II study: a randomized controlled trial. Prolyse in Acute Cerebral Thromboembolism. JAMA (1999) 282:2003-11. doi:10.1001/jama.282.21.2003

20. Pexman JH, Barber PA, Hill MD, Sevick RJ, Demchuk AM, Hudon ME, et al. Use of the Alberta stroke program early CT score (ASPECTS) for assessing CT scans in patients with acute stroke. AJNR Am J Neuroradiol (2001) 22:1534-42.

21. Schramm P, Schellinger PD, Klotz E, Kallenberg K, Fiebach JB, Kulkens S, et al. Comparison of perfusion computed tomography and computed tomography angiography source images with perfusion-weighted imaging and diffusion-weighted imaging in patients with acute stroke of less than 6 hours' duration. Stroke (2004) 35:1652-8. doi:10.1161/01.STR.0000131271.54098.22

22. Maija R, Gaida K, Karlis K, Evija M. Perfusion computed tomography relative threshold values in definition of acute stroke lesions. Acta Radiol Short Rep (2013) 2:2047981613486099. doi:10.1177/2047981613486099

23. Wintermark M, Flanders AE, Velthuis B, Meuli R, van Leeuwen M, Goldsher D, et al. Perfusion-CT assessment of infarct core and penumbra: receiver operating characteristic curve analysis in 130 patients suspected of acute hemispheric stroke. Stroke (2006) 37:979-85. doi:10.1161/01.STR.0000209238.61459.39

24. Coutts SB, Lev MH, Eliasziw M, Roccatagliata L, Hill MD, Schwamm LH, et al. ASPECTS on CTA source images versus unenhanced CT: added value in predicting final infarct extent and clinical outcome. Stroke (2004) 35:2472-6. doi:10.1161/01.STR.0000145330.14928.2a

25. Fazekas F, Chawluk JB, Alavi A, Hurtig HI, Zimmerman RA. MR signal abnormalities at $1.5 \mathrm{~T}$ in Alzheimer's dementia and normal aging. AJR Am J Roentgenol (1987) 149:351-6. doi:10.2214/ajr.149.2.351

26. Adams HP Jr, Bendixen BH, Kappelle LJ, Biller J, Love BB, Gordon DL, et al. Classification of subtype of acute ischemic stroke. Definitions for use in a multicenter clinical trial. TOAST. Trial of Org 10172 in acute stroke treatment. Stroke (1993) 24:35-41. doi:10.1161/01.STR.24.1.35

27. Wardlaw JM, Smith EE, Biessels GJ, Cordonnier C, Fazekas F, Frayne R, et al. Neuroimaging standards for research into small vessel disease and its contribution to ageing and neurodegeneration. Lancet Neurol (2013) 12:822-38. doi:10.1016/S1474-4422(13)70124-8

28. Barber PA, Demchuk AM, Zhang J, Buchan AM. Validity and reliability of a quantitative computed tomography score in predicting outcome of hyperacute stroke before thrombolytic therapy. ASPECTS Study Group. Alberta stroke programme early CT score. Lancet (2000) 355:1670-4. doi:10.1016/ S0140-6736(00)02237-6

29. Grotta JC, Chiu D, Lu M, Patel S, Levine SR, Tilley BC, et al. Agreement and variability in the interpretation of early CT changes in stroke patients qualifying for intravenous rtPA therapy. Stroke (1999) 30:1528-33. doi:10.1161/ 01.STR.30.8.1528

30. Jain AR, Jain M, Kanthala AR, Damania D, Stead LG, Wang HZ, et al. Association of CT perfusion parameters with hemorrhagic transformation in acute ischemic stroke. AJNR Am J Neuroradiol (2013) 34:1895-900. doi:10.3174/ajnr.A3502

31. Campbell BC, Christensen S, Parsons MW, Churilov L, Desmond PM, Barber PA, et al. Advanced imaging improves prediction of hemorrhage 
after stroke thrombolysis. Ann Neurol (2013) 73:510-9. doi:10.1002/ ana. 23837

32. Molina CA, Montaner J, Abilleira S, Ibarra B, Romero F, Arenillas JF, et al. Timing of spontaneous recanalization and risk of hemorrhagic transformation in acute cardioembolic stroke. Stroke (2001) 32:1079-84. doi:10.1161/ 01.STR.32.5.1079

33. Sussman ES, Connolly ES Jr. Hemorrhagic transformation: a review of the rate of hemorrhage in the major clinical trials of acute ischemic stroke. Front Neurol (2013) 4:69. doi:10.3389/fneur.2013.00069

34. Schaefer PW, Souza L, Kamalian S, Hirsch JA, Yoo AJ, Kamalian S, et al. Limited reliability of computed tomographic perfusion acute infarct volume measurements compared with diffusion-weighted imaging in anterior circulation stroke. Stroke (2015) 46:419-24. doi:10.1161/STROKEAHA. 114.007117

35. Kamalian S, Kamalian S, Maas MB, Goldmacher GV, Payabvash S, Akbar A, et al. CT cerebral blood flow maps optimally correlate with admission diffusion-weighted imaging in acute stroke but thresholds vary by postprocessing platform. Stroke (2011) 42:1923-8. doi:10.1161/STROKEAHA. 110.610618

36. Campbell BC, Christensen S, Levi CR, Desmond PM, Donnan GA, Davis SM, et al. Cerebral blood flow is the optimal CT perfusion parameter for assessing infarct core. Stroke (2011) 42:3435-40. doi:10.1161/STROKEAHA.111.618355
37. Thierfelder KM, Sommer WH, Baumann AB, Klotz E, Meinel FG, Strobl FF, et al. Whole-brain CT perfusion: reliability and reproducibility of volumetric perfusion deficit assessment in patients with acute ischemic stroke. Neuroradiology (2013) 55:827-35. doi:10.1007/s00234-013-1179-0

38. van Seeters T, Biessels GJ, Niesten JM, van der Schaaf IC, Dankbaar JW, Horsch AD, et al. Reliability of visual assessment of non-contrast CT, CT angiography source images and CT perfusion in patients with suspected ischemic stroke. PLoS One (2013) 8:e75615. doi:10.1371/journal.pone.0075615

Conflict of Interest Statement: The authors declare that the research was conducted in the absence of any commercial or financial relationships that could be construed as a potential conflict of interest.

The reviewer NY and handling editor declared their shared affiliation.

Copyright (c) $2017 \mathrm{Liu}$, Wu, Zhao, Cao, Dedhia, Caplan and Wang. This is an open-access article distributed under the terms of the Creative Commons Attribution License (CC BY). The use, distribution or reproduction in other forums is permitted, provided the original author(s) or licensor are credited and that the original publication in this journal is cited, in accordance with accepted academic practice. No use, distribution or reproduction is permitted which does not comply with these terms. 